\section{Investigation of osteogenesis changes in medaka larvae reared in normal gravity, simulated- microgravity and hypergravity environments}

\author{
Natsuhiro Takahashi ${ }^{\mathrm{a}}$, Masamichi Takami ${ }^{\mathrm{a}, \mathrm{b}}$, \\ Masahiro Chatani ${ }^{\mathrm{a}, \mathrm{b}_{*}}$ \\ ${ }^{a}$ Pharmacological Research Center, Showa \\ University, Tokyo 142-8555, Japan \\ ${ }^{b}$ Department of Pharmacology, Showa University \\ School Dentistry, Tokyo 142-8555, Japan
}

\begin{abstract}
Bones are important organs for body resistance against force produced by gravity, though the influence of gravity on bone development is unclear. To examine the effects of gravity on osteogenesis, medaka larvae were reared in water or gel under various conditions. For determining the effects on bone development in a state of motion, larvae were reared in water under normal gravity $(1 \mathrm{~g})$ or hypergravity $(5 \mathrm{~g})$ conditions. Also, to examine the direct effect of gravity on bone mineralization, larvae were embedded in low melting agarose gel containing alizarin complexone (ALC) and reared for three days under a normal gravity (1g), simulated-microgravity $(s-\mu g)$ with use of a clinostat device, or $5 g$ condition. Medaka reared in water under the $5 g$ condition showed forward protruding jaws and spreading of the mineralized area of jaw teeth as compared to those reared under the $1 \mathrm{~g}$ control condition. In addition, the direction of growth of the notochord in the fin region was changed upward in those reared under the $5 \mathrm{~g}$ condition, accompanied by a part of acetylated tubulinpositive nerves also localized upward, while positive signals for DsRed, expressed by an osterix promoter, in osteoblasts were increased in the fin region. On the other hand, in medaka reared in gel, ALC signals in the fin ray of those in the $s-\mu g$ condition were increased as compared to those in the $5 \mathrm{~g}$ condition. Changes noted in medaka larvae over three days indicated osteogenesis adaptation to the specific gravity environment. The present results obtained with an experimental system
\end{abstract}

Received: July 9, 2021; Accepted: August 6, 2021

*To whom Correspondence should be addressed:

Masahiro Chatani, Department of Pharmacology, School of

Dentistry, Showa University, Tokyo 142-8555, Japan

Tel.: +81-3-3784-8175, Fax: +81-3-3784-8176,

Email: chatani@dent.showa-u.ac.jp are considered useful for examinations in the future regarding changes of osteogenesis, which will be needed to clarify the mechanism of the effects of gravity on bone development. O2021 Jpn. Soc. Biol. Sci. Space; doi:10.2187/bss.35.24

\section{Introduction}

During space flight, changes in bones under a microgravity condition are dependent on the skeletal-site position relative to the gravitational vector. (Stavnichuk et al. 2020). For the various vertebrates present on Earth, bones are important organs that support body weight produced by gravity, though it not clears how gravity affects bone development.

Small fish, such as medaka and zebrafish, provide a good animal model for examining hard tissues under various gravity environments, such as microgravity in a space environment, simulated-microgravity $(s-\mu g)$ generated by a clinostat device, and hypergravity. In 1994, medaka were transported to space in a space shuttle, and the first investigations of mating and rearing under microgravity were performed (ljiri 1995). In 2012, osteoclast- and osteoblast-double transgenic medaka lines were reared for two months at the International Space Station (ISS) for a study of bone metabolism under microgravity (Chatani et al. 2015), while changes in fluorescent signals in bone-related transgenic medaka larvae embedded in gel at the ISS were also observed (Chatani et al. 2016). On the other hand, clinostat devices have been used for examinations of the effects of microgravity on earth, as it has an ability to cancel the sum of the gravity vector as a time average by changing its direction (Borst and Van Loon 2009). Another study reported bone changes in zebrafish larvae reared in clinostats in water rather than gels (Aceto et al. 2016), while in our previous examination of hypergravity, young adult medaka were reared under a $5 g$ condition for six months, which resulted in findings showing spine curvature and otolith formation defects (Chatani et al. 2019). More recently, it was reported that hypergravity $(6 g)$ induced abnormal chondrocyte formation in zebrafish larvae (Lawrence et al. 2021). However, the involvement of gravity in bone development remains to be elucidated.

The present study was conducted to assess bone morphology in larvae reared in water under $5 g$ and $1 g$ (control) conditions. In addition, signals showing uptake of alizarin complexone (ALC) in larvae reared in gel under $s-\mu g$, hypergravity $(5 g)$, control $(1 g)$ conditions were also examined. The results showed several differences in bone formation depending on the gravity condition. We consider that this study will be useful for providing base research findings regarding gravity-related bone biology.

\section{Materials and Methods}

\section{Egg collection}

Eggs were obtained from $\mathrm{Cab}$ or TRAP promoterGFP/osterix promoter-DsRed double transgenic medaka lines, and placed in 30-mm dishes (SUMILON Dish, Sumitomo Bakelite Co., Ltd.) filled with egg water, and 
then shaken for seven days on a shaker (Labo Shaker BC-730, Bio Craft) in a $28^{\circ} \mathrm{C}$ incubator (MIR-154S-PJ, Panasonic).

\section{Rearing larvae in water}

Hatched medaka larvae from Cab or TRAP-GFP/ Osterix-DsRed double transgenic lines were placed in bottles (Nunc EasyFlask $75 \mathrm{~cm}^{2}$, Thermo Scientific) containing $200 \mathrm{ml}$ of rearing water, then in an incubator for three days with light blocked. For the $5 g$ condition, that was generated by spinning with a centrifuge (LIX-140SP, Tomy Seiko Co., Ltd) at $160 \mathrm{rpm}$ (Chatani et al. 2019).

\section{Alizarin Red stain}

Medaka were fixed in 4\% Paraformaldehyde Phosphate Buffer Solution (PFA; Fujifilm Wako Pure Chemical Corporation) overnight at $4^{\circ} \mathrm{C}$, then washed with PBS and placed into $0.1 \mathrm{~N} \mathrm{KOH}$. Next, $50 \mu \mathrm{l}$ of Alizarin Red solution saturated in ethanol was added to $1 \mathrm{ml}$ of $0.1 \mathrm{~N} \mathrm{KOH}$, mixed gently, and allowed to stand overnight at $4^{\circ} \mathrm{C}$. After rinsing with $0.1 \mathrm{~N} \mathrm{KOH}$, the samples underwent stepwise replacement with Glycerin (Fujifilm Wako Pure Chemical Corporation) $70 \% / 0.1 \mathrm{~N}$ $\mathrm{KOH}$ and photographed under a microscope.

\section{Nerve stain}

Larvae were fixed with $80 \%$ methanol/20\% DMSO overnight at $4^{\circ} \mathrm{C}$. After washing three times with $1 \mathrm{ml}$ of PBST $(0.1 \%$ Tween), $10 \%$ sheep serum/PBST at $200 \mu \mathrm{l}$ was used as the blocking liquid. Next, monoclonal antiacetylated tubulin antibodies (Sigma, T7451) at $0.1 \mu \mathrm{l}$ were added as the primary antibodies and the larvae were left overnight at $4^{\circ} \mathrm{C}$, then washing with $1 \mathrm{ml}$ of PBST was performed three times and Donkey Anti-Mouse IgG H\&L (Alexa Fluor $^{\circledR}$ 568, Abcam, ab175472) at $0.1 \mu \mathrm{l}$ was added and again they were left overnight at $4^{\circ} \mathrm{C}$. Finally, fins were washed using PBS.

\section{Rearing larvae in agarose gel}

Medaka larvae were anesthetized at two days after hatching by placing them on ice and then into a $30-\mathrm{mm}$ dish that had been previously filled with $4 \mathrm{ml}$ of low melting point agarose gel (Agarose-LM Sieve, Nacalai Tesque). Next, another $4 \mathrm{ml}$ of liquid low melting point agarose gel was added and the specimen was oriented with tweezers. The agarose gel was prepared in autoclaved rearing water containing $1 \%$ alizarin complexone (ALC; 348-00093, Wako Pure Chemical Corporation).

Each dish was set under the following gravity conditions with no light. The $1 \mathrm{~g}$ control group was placed stationary on a desk. For the $5 g$ (5.29 times greater than normal gravity) group, that was generated by centrifuge spinning at $160 \mathrm{rpm}$ (Chatani et al. 2019), while the $s-\mu g$ group was rotated in a clinostat device (Zeromo CL-100, Kitagawa). Larvae were removed from the agarose gel after three days and signals indicating calcification were observed using a fluorescence microscope (M205FA, Leica).

\section{Measurements}

All images were captured using LAS $X$ software (LAS
$X$ 3.3.169258.3, Leica) and body part lengths were measured with another LAS $X$ software package (LAS X 3.7.21655.1, Leica). Area and intensity of fluorescence in the images of medaka larvae were determined by use of Fiji ImageJ software (ImageJ 1.53c, NIH) (Schneider, Rasband, and Eliceiri 2012). All graphs were constructing with RStudio Desktop, v. 1.4.1717.

For quantitative analysis of mandibular protrusion to the maxilla (lateral) (Fig. 2C), measurement values were calculated using an original formula for normalization, as shown following. Head=p, Mandibular=q, Body length=L, Mandibular protrusion to maxilla (lateral $)=\frac{\frac{q}{p}}{L}$.

For quantitative analysis of mandibular protrusion (dorsal) (Fig. 2F), measurement values were calculated using an original formula for normalization, as shown following. Head=x, Mandibular=y, Body length=L, Mandibular protrusion (dorsal) $=\frac{\frac{y}{x}}{L}$.

For quantitative analysis of cross eye distance (Fig. $2 \mathrm{~F}$ ), measurement values were calculated using an original formula for normalization, as shown following. Right eye $=\mathrm{v}$, Left eye $=\mathrm{w}$, Eye distance $=\mathrm{z}$, Body length $=\mathrm{L}$,

Cross eye distance $=\frac{\frac{\mathrm{z}}{\left(\frac{\mathrm{v}+\mathrm{w}}{2}\right)}}{\mathrm{L}}$.

For quantitative analysis of the aspect ratio of the spine (Fig. 2J), measurement values were calculated using an original formula for normalization, as shown following. $13^{\text {th }}$ Width $=\mathrm{I}, 13^{\text {th }}$ Length $=\mathrm{m}, 14^{\text {th }}$ Width $=\mathrm{n}, 14^{\text {th }}$ Length $=0$, Body length $=L$, Aspect ratio of the spine $=\frac{\frac{\frac{l}{m}+\frac{n}{o}}{2}}{L}$.

For quantitative analysis of mandibular protrusion (dorsal) (Fig. 5C), measurement values were calculated using an original formula for normalization, as shown following. Head=x, Mandibular=y, Body length=L, Mandibular protrusion (dorsal) $=\frac{\frac{y}{x}}{L}$.

For quantitative analysis of the aspect ratio of the spine (Fig. 5E), measurement values were calculated using an original formula for normalization, as shown following. $14^{\text {th }}$ Width $=1,14^{\text {th }}$ Length $=\mathrm{m}, 15^{\text {th }}$ Width $=\mathrm{n}, 15^{\text {th }}$ Length $=0$, Body length $=L$, Aspect ratio of the spine $=\frac{\frac{\frac{1}{m}+\frac{n}{o}}{2}}{L}$.

For quantitative analysis of the growth area of the 
spine (Fig. 5G), measurement values were calculated using an original formula for normalization, as shown following. Upper front=p, Upper rear=q, Lower front=r, Lower rear=s, Body length=L, Growth area of the spine= $\frac{p+q+r+s}{L}$. (Total area from $15^{\text {th }}$ to $18^{\text {th }}$.)

\section{Statistics}

Calculated values for the $1 g, s-\mu g$, and $5 g$ conditions were compared, and p-values were estimated using Tukey's test. Graph data are mean \pm s.d.

\section{Results}

Medaka larvae are relatively small in size and bone formation through the whole body can be easily observed. To examine the effects of gravity in a state of motion while in water, hatched larvae were reared in water for three days under the $1 g$ or $5 g$ condition, then bones were stained with Alizarin Red (Fig. 1A, B). Those results showed a significant difference in body length between the gravity conditions (Fig. 2A, B). Observations of heads from the lateral or dorsal side showed that the mandible protruded forward in the $5 \mathrm{~g}$ group as compared to the $1 \mathrm{~g}$ group (control) (Fig. 2C-F). Furthermore, observations in those two directions revealed increases in mandibular teeth and bone areas in the $5 g$ group (Fig. $2 \mathrm{C}, \mathrm{F}-\mathrm{H}$ ), while there was no difference in distance between the eyes (Fig. 2F, I), suggesting that the mandible specifically was deformed as a result of higher gravity. The ratio of length to width of the vertebrae in the lateral view (Fig. 2J, K) was unchanged. In the fin region, the notochord was bent upward at a greater angle in larvae reared in the $5 g$ environment as compared to the control group (Fig. 2L, M). Also, acetylated tubulin staining showed that some of the nerves were localized upward along the notochord in medaka reared in the higher gravity condition (Fig. 3A). In the $5 g$ group, the number of cells positive for TRAP, a known osteoclast marker, was decreased (Fig. 3E, G), whereas the intensity of osterix-DsRed signals in osteoblasts was increased (Fig. 3B, D). There were no differences between the groups regarding fluorescence areas of osterix-DsRed and TRAP-GFP (Fig. 3C, F). These findings indicated that the properties of cells constituting fin tissue were changed according to the gravitational environment.

Next, to examine the direct effects of gravity on bone growth, larvae were embedded in low-melting-point agarose gel containing alizarin complexone (ALC), which marks calcium uptake on the bone surface during bone formation (Fig. 4A). Thereafter, larvae at two days after hatching were reared for three days in a $1 \mathrm{~g}$ (normal gravity), $s-\mu g$ (Fig. 4B), or $5 g$ (Fig. 4C) environment. Our results showed no significant differences in morphology, such as body length, ratio of jaw protrusion, spine shape, or bending of the notochord in the fin region (Fig. 5A-J). At the end of the spine, the area with ALC fluorescence signals tended to be greater under the $s-\mu g$ condition as compared to the others, indicating increased uptake. Although, though the differences were not significant, a comparison between the $s-\mu g$ and $5 g$ groups showed a p-value of 0.173 (Fig. 5G, H). Furthermore, while the area of ALC fluorescence signals in the fin ray did not change, the intensity of ALC fluorescence signals there was increased under the $s-\mu g$ as compared to the $5 g$ condition (Fig. 5K-M).

\section{Discussion}

Using a novel approach to better understand the involvement of gravity in osteogenesis, some striking changes were noted in larvae. We have investigated bone formation under two conditions. One is rearing in water, the condition that is affected by floating power. The other is rearing in a gel, which we consider as a kind of organ culture, which is not affected by floating power. A clinostat is a device that uses rotation to cancel the sum of gravity vectors over a period of time. The condition in this device

A

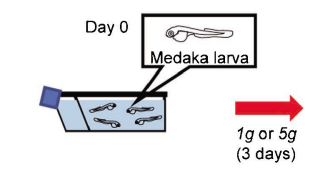

Hatching larvae in bottle

B

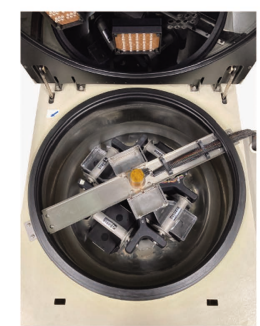

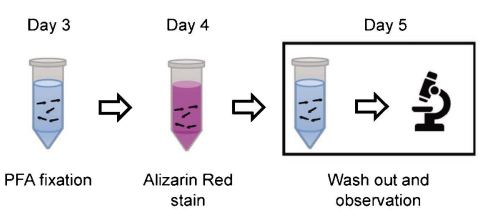

observation

Figure 1. Overview of bone development analysis using larvae reared in water under $1 g$ and $5 g$ conditions.

(A) Schedule for bone staining of larvae reared for three days in $1 g$ and $5 g$. (B) Bottles placed in centrifuge. 
A

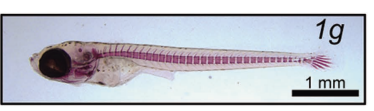

C
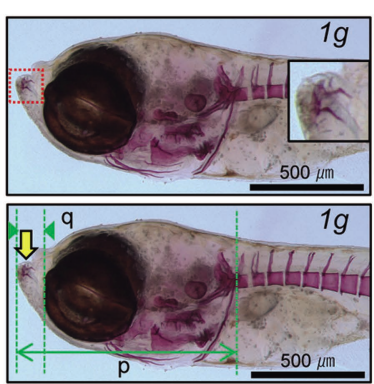

$\mathrm{F}$
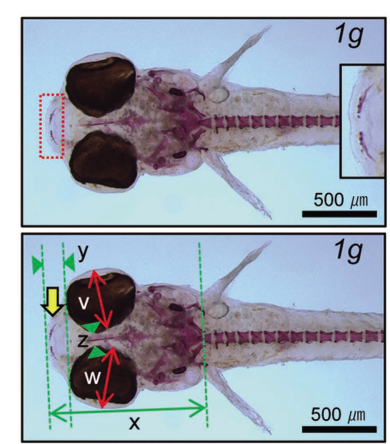

J

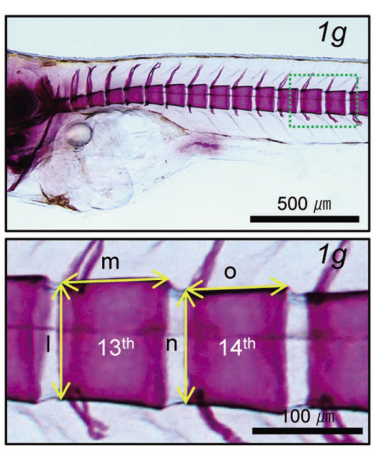

L

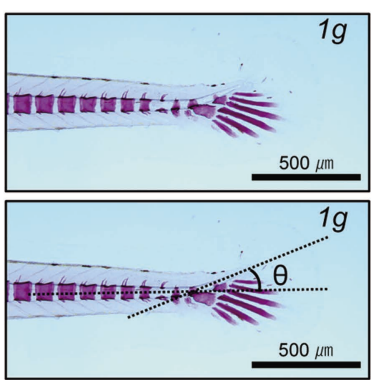

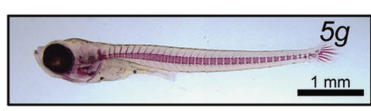
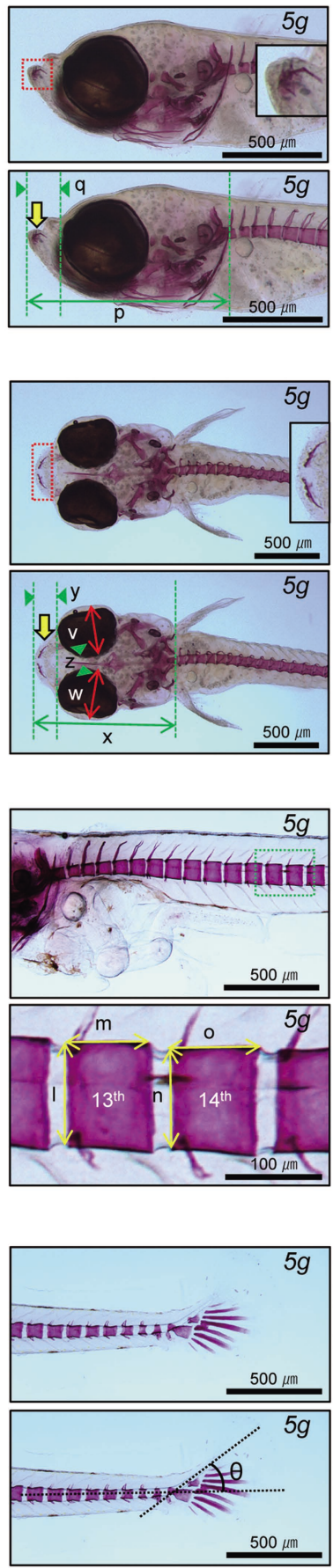

B

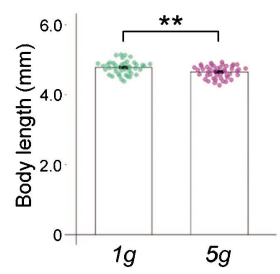

D
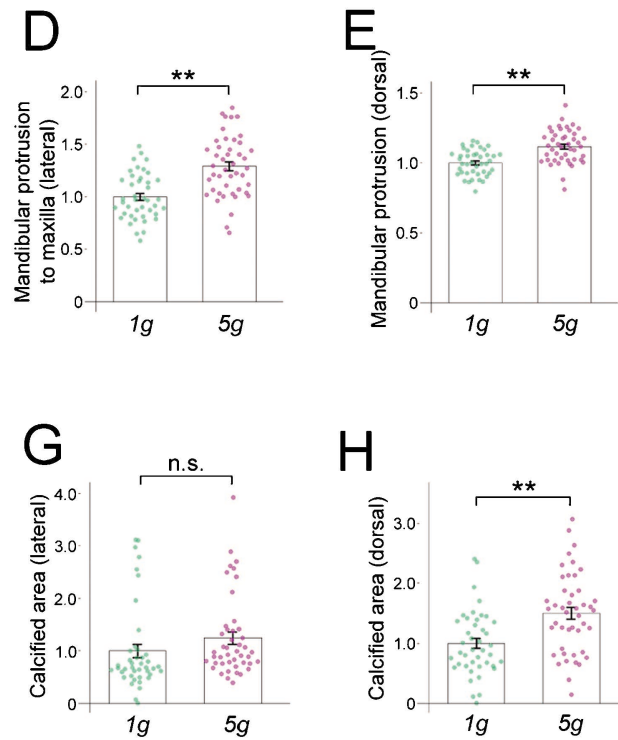

$\mathrm{H}$

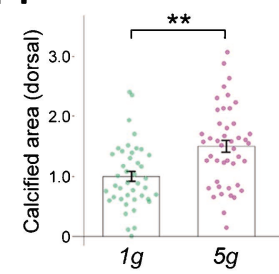

$\mathrm{K}$
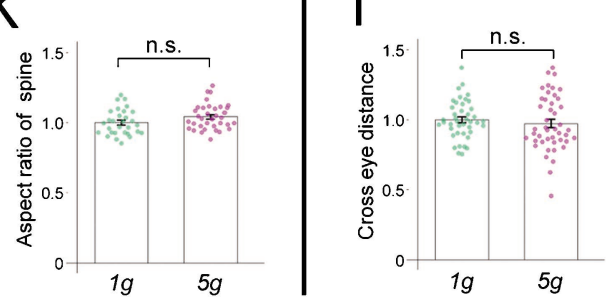

M

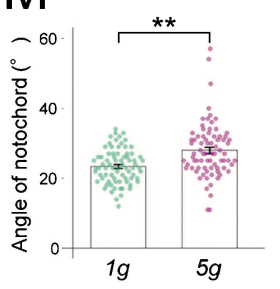

Figure 2. Hypergravity in state of motion in water affects jaw protrusion and fin formation.

Alizarin Red staining of medaka larvae reared under $1 g$ and $5 g$ conditions for three days. (A) Lateral views of whole body. (B) Comparison of body length. (C) Lateral view of head region (upper) and measured sites (lower). p, q: measured length. Yellow arrows show lower jaw teeth. (D) Comparison of mandibular protrusion to maxilla (lateral). (E) Comparison of mandibular protrusion (dorsal). (F) Dorsal view of head region (upper) and measured sites (lower). v, w, x, y, z: measured length. Yellow arrows show jaw teeth. (G) Comparison of calcified area (lateral). (H) Comparison of calcified area (dorsal). (I) Comparison of cross eye distance. (J) Lateral view of vertebral body (upper). Enlarged views show $13^{\text {th }}$ and $14^{\text {th }}$ vertebrae in dotted squares indicated in upper images (lower). I, m, n, $\mathrm{o}$ : measured length. (K) Comparison of aspect ratio of spine. (L) Lateral view of fin region (upper). $\Theta$ in lower images show angle of bending notochord. (M) Comparison of angle of notochord. P-values were determined using Tukey's test. n.s.: not significant difference, ${ }^{* *} \mathrm{p}<0.01$. (B, D, E, G, H, I: 1g, n=42; 5g, n=45. K: 1g, n=30; 5g, n=35. M: 1g, n=76; 5g, n=77) 

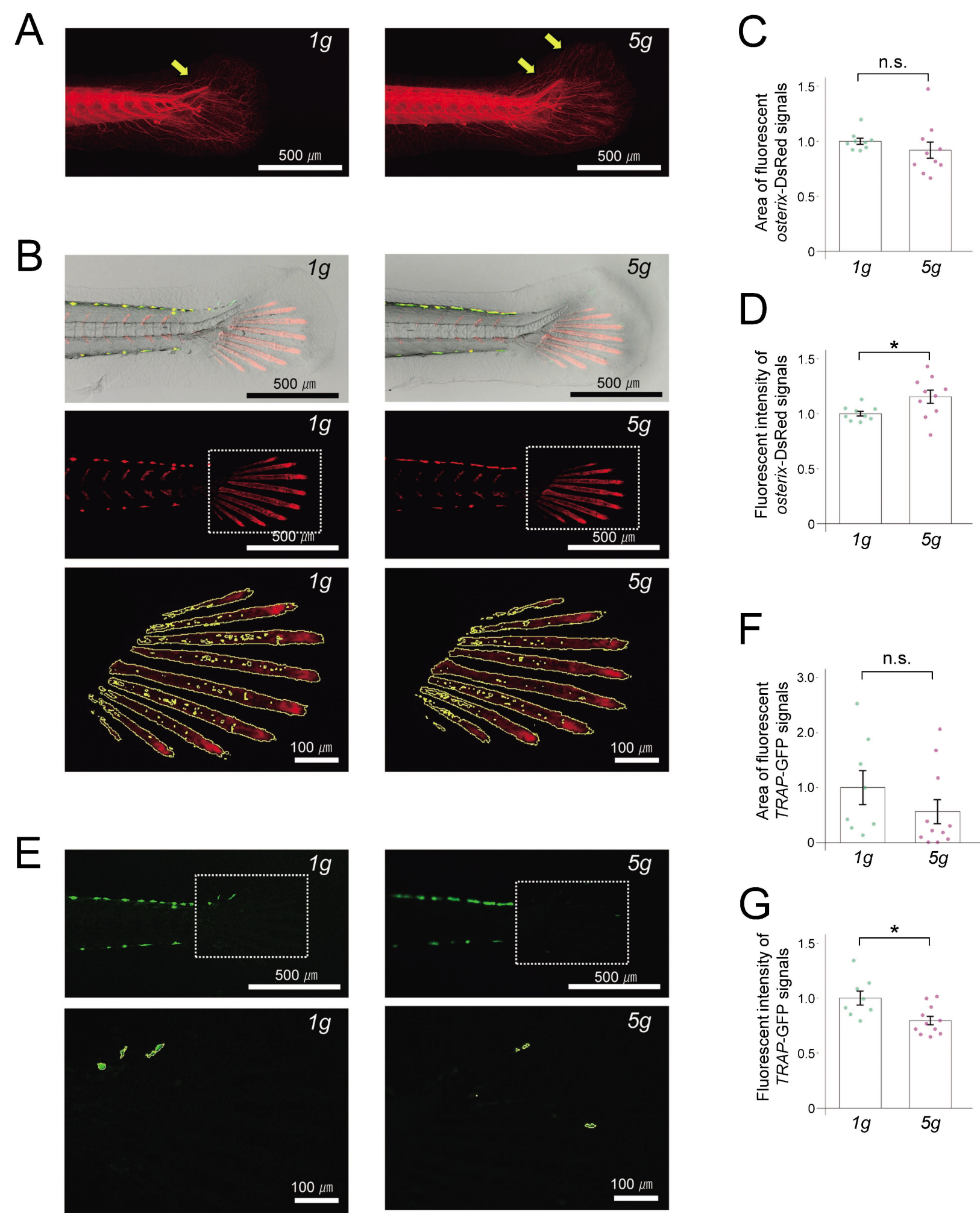

$\mathrm{F}$

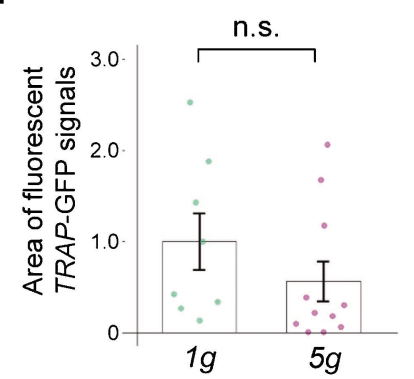

$G$

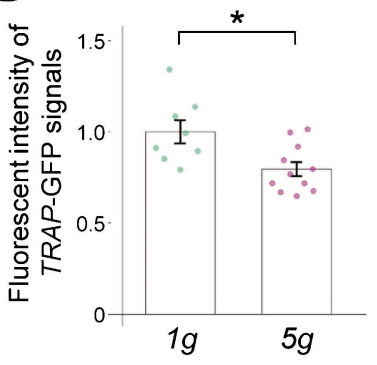

Figure 3. Distribution of nerve and osteoblast changes in larvae reared in water under hypergravity.

Comparison of fin region in larvae reared for three days under $1 \mathrm{~g}$ and $5 \mathrm{~g}$ conditions. (A) Acetylated tubulin staining. (B-G) Analysis of osterix-DsRed/TRAP-GFP double-stained transgenic medaka line. (B) Merged images of optical and osterix-DsRed/TRAP-GFP (upper), and osterix-DsRed (middle and lower) signals. Area circled in yellow in lower images shows measurement area. (C) Comparison of areas of fluorescent osterix-DsRed signals. (D) Comparison of fluorescent intensity of osterix-DsRed signals. (E) TRAP-GFP. Area circled in yellow in lower images shows measurement area. (F) Comparison of areas of fluorescent TRAP-GFP signals. (G) Comparison of areas of fluorescent intensity of TRAP-GFP signals. P-values were determined using Tukey's test. n.s.: not significant difference, ${ }^{\star} p<0.05$. (C, D: $1 g, \mathrm{n}=9 ; 5 g, \mathrm{n}=10 . \mathrm{F}, \mathrm{G}: 1 g, \mathrm{n}=8 ; 5 g, \mathrm{n}=11$.) 

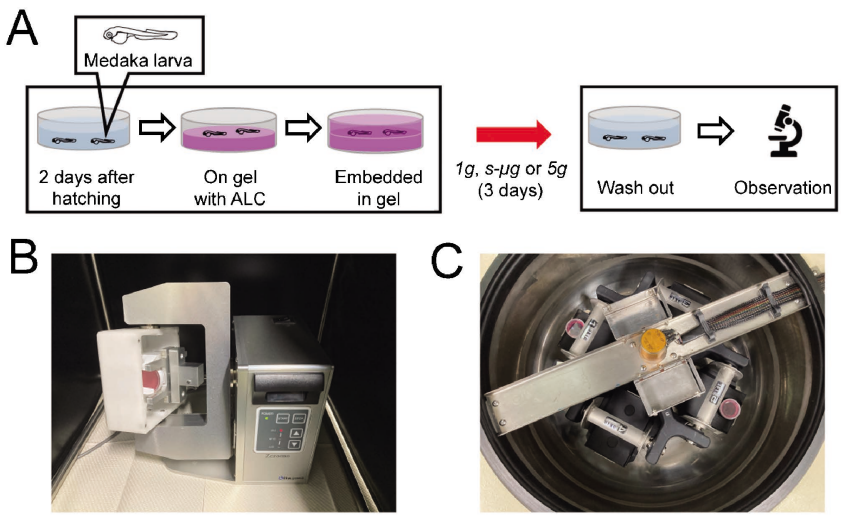

Figure 4. Overview of ALC uptake analysis using larvae embedded in agarose gel under $1 g, s-\mu g$, and $5 g$ conditions.

(A) Schedule for bone staining and gravity change. Larvae were stained with alizarin complexone (ALC) and kept under one of the three gravity conditions for three days. (B) Dish placed in clinostat device for $s-\mu g$ condition. (C) Dish placed in centrifuge for $5 g$ condition.

was defined as a simulated-microgravity condition $(s-\mu g)$, and the effect on bone formation in larvae embedded in gel was investigated.

For medaka kept in water, changes were observed in two different areas. First, the jaw protruded forward and the calcified area was increased in those reared in the $5 \mathrm{~g}$ environment. Other studies have reported that mandibles in mice and rats changed under various gravity conditions, indicating that mandibular formation may be dependent on gravity (Ghosh et al. 2016; Mednieks and Hand 2019; Simmons et al. 1983). In addition, as dentistry is considered to be an important issue for astronauts living in space (Zaitsu et al. 2014), it is expected that the related mechanism will be clarified in the future. Additionally, the present results showed that the fins grew upward in medaka exposed to the hypergravity environment. The tail fin has been shown to have an optimal shape (Zhiqiang and Chuijie 2013), with its structure remodeled according to specific types of fin motion (Enny et al. 2020) and the angle of the caudal fin having an effect on thrust production (Matta et al. 2019). Thus, the present finding of an upward-facing fin phenotype may indicate that the morphology of the medaka fin has an ability to change to adapt to a hypergravity environment.

Together, the gravity preferentially affected structure of the jaw and the fin. The mandible is located below the maxilla and is the only bone in the skull that can be moved. Since the mandible is located where it needs to resist the direction of gravity, the mandible may be the sensitive tissue to the effects of gravity. It is thought that floating power may be insufficient for larvae to swim in a $5 g$ environment, and it is inferred that the fin structure was changed to move it in order to increase the lift force. As for the body length, the jaws were slightly longer in $5 g$, but the body length was shorter because the fins were bent farther upward.

When reared in gel, medaka in the $s-\mu g$ condition tended to show greater amounts of uptake of ALC into the vertebral body and fin ray as compared to those in the $5 \mathrm{~g}$ condition. Other studies have noted changes in osteoblast activity under clinostat conditions (Granet et al. 2002;
Ulbrich et al. 2014), suggesting that gravity vector diffusion stimulates medaka osteoblasts and promotes bone formation.

The results of our experiments with medaka reared in water indicate that the composition of fins and teeth changed as a result of adaptation to movement in a hypergravity environment. On the other hand, results of those reared in gel seem to show that gravity had direct effects on calcification, because the medaka were unable swim. These results provide an initial step in understanding gravity-dependent osteogenesis. We did not confirm any changes in the osteoclasts on vertebrae because we observed them at an early developmental stage. In future studies, detailed analysis of related mechanisms is anticipated to provide new discoveries, including changes in gene expression levels under various gravity conditions.

\section{Acknowledgements}

We thank all the members of our laboratory for their insightful comments and suggestions. We gratefully acknowledge Dr. Akira Kudo of Tokyo Institute of Technology for providing the experimental equipment, Dr. Junichi Tanaka of Showa University for support regarding the use of Rstudio, and Dr. Yuki Azetsu of Showa University for the helpful advice. The experiments were performed in accordance with policies and protocols approved by the Institutional Animal Care and Use Committee of Showa University. This work was supported in part by a Grant-in-Aid for Scientific Research (C) to M. C. (No. 21K06255), a Grant-in-Aid for Scientific Research on Innovative Areas to M. C. (No. 18H04986), and a Fostering Joint International Research (B) grant to M. C. (No. 19KK0233) from the Japan Society for the Promotion of Science, as well as grants-in aid from the Moritani Scholarship Foundation to M. C and grants-in-aid from the Ministry of Education, Culture, Sports, Science and Technology of Japan.

\section{Authors' contributions}

M.C. designed the research contents, supported the 
A

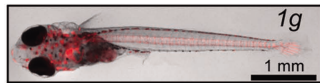

C

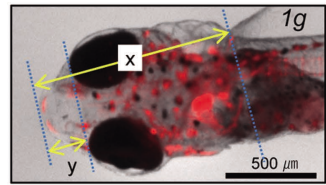

$\mathrm{E}$

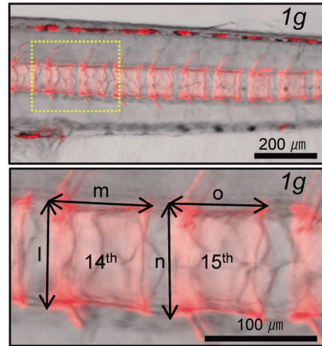

G
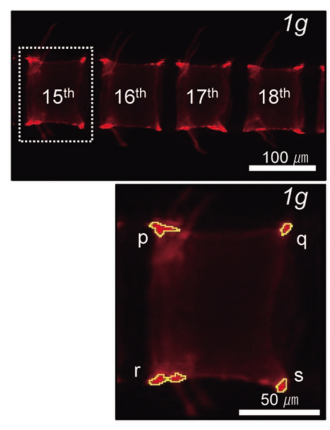

|
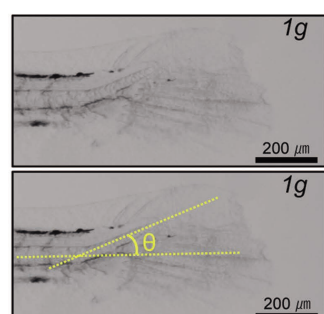

$\mathrm{K}$

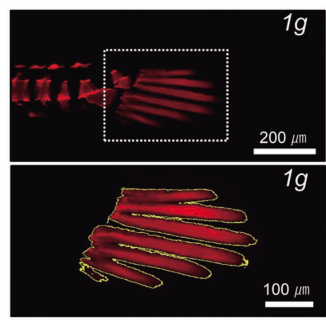

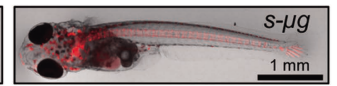
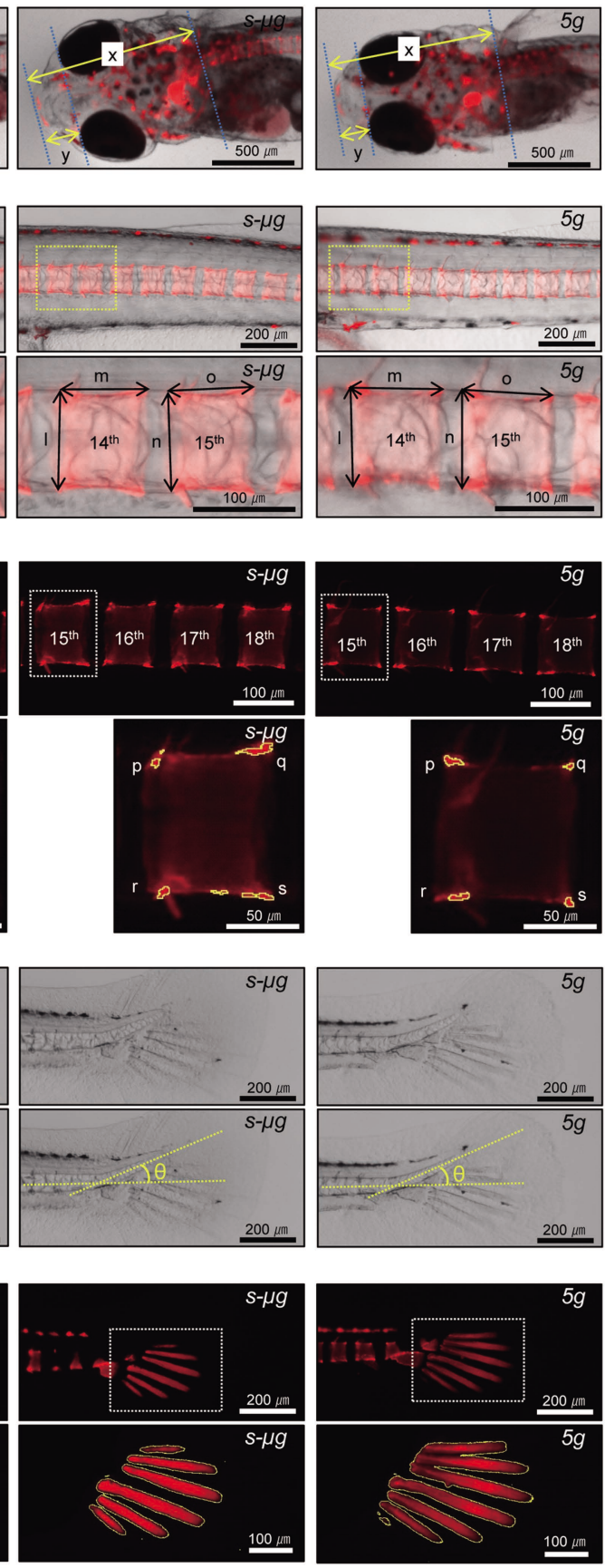
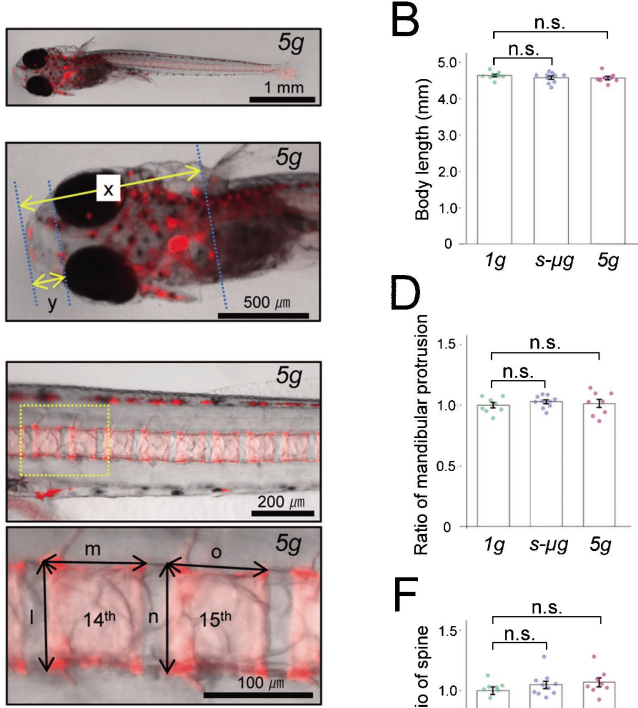

D

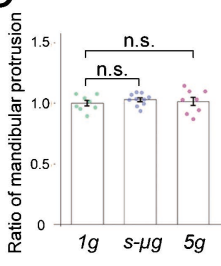

F
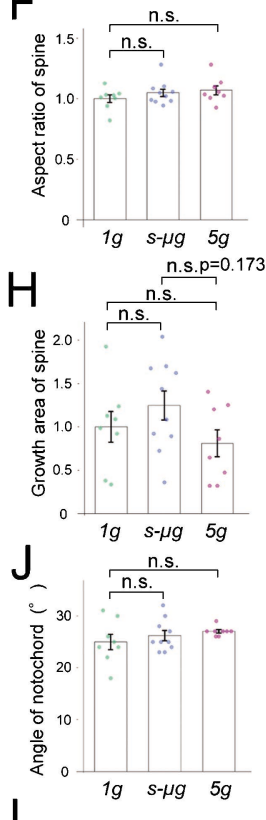

L
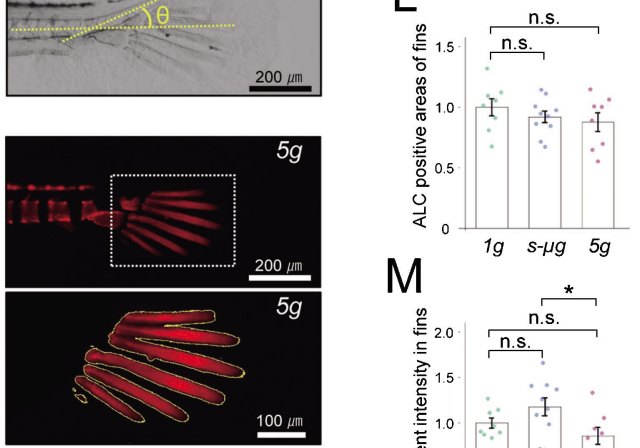

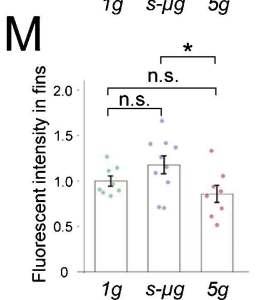

Figure 5. ALC uptake increased in spine and fin ray under $s-\mu g$ condition.

Larvae were kept under $1 g, s-\mu g$, and $5 g$ conditions, and stained with alizarin complexone (ALC). (A) Whole body. (B) Comparison of body length. (C) Enlarged view of head region. $x, y$ : measured lengths. (D) Comparison of ratio of mandibular protrusion. (E) Lateral view of vertebral body (upper). Enlarged views show $14^{\text {th }}$ and $15^{\text {th }}$ vertebrae in dotted squares indicated in upper images (lower). I, $\mathrm{m}, \mathrm{n}, \mathrm{O}$ : measured lengths. (F) Comparison of aspect ratio of spine. (G) ALC signals in vertebral body. Enlarged view shows measured area to determine epiphyseal bone growth. $(\mathrm{H})$ Growth area of spine. A comparison of the $s-\mu g$ and $5 g$ conditions showed a $p$-value of 0.173 . (I) Optical image of fin region. $\Theta$ in lower images shows angle of bending notochord. (J) Comparison of angle of notochord. (K) ALC signals in fin region. Area circled in yellow in lower images shows measurement area. (L) Comparison of ALC positive areas of fins. (M) Comparison of fluorescent intensity in fins. P-values were determined using Tukey's test. n.s.: not significant difference, ${ }^{*} p<0.05$. (B, D, F, H, J, L, M: $1 g, \mathrm{n}=8 ; s-\mu g, \mathrm{n}=10 ; 5 g, \mathrm{n}=8$.) 
experiments, and wrote the manuscript. N.T. performed the experiments, summarized the results, and performed quantitative analysis. M.T. provided the research environment and advised regarding the content of the paper.

\section{Declaration of Interests}

The authors have no competing financial interests to declare.

\section{References}

Aceto, Jessica, Rasoul Nourizadeh-Lillabadi, Silvia Bradamante, Jeanette A. Maier, Peter Alestrom, Jack JWA van Loon, and Marc Muller. 2016. "Effects of Microgravity Simulation on Zebrafish Transcriptomes and Bone Physiology-Exposure Starting at 5 Days Post Fertilization." Npj Microgravity 2(1): 16010.

Borst, A. G., and Jack J. W. A. Van Loon. 2009. "Technology and Developments for the Random Positioning Machine, rpm." Microgravity Science and Technology 21(4):287-92.

Chatani, Masahiro, Akiko Mantoku, Kazuhiro Takeyama, Dawud Abduweli, Yasutaka Sugamori, Kazuhiro Aoki, Keiichi Ohya, Hiromi Suzuki, Satoko Uchida, Toru Sakimura, Yasushi Kono, Fumiaki Tanigaki, Masaki Shirakawa, Yoshiro Takano, and Akira Kudo. 2015. "Microgravity Promotes Osteoclast Activity in Medaka Fish Reared at the International Space Station." Scientific Reports 5(14172): 1-13.

Chatani, Masahiro, Aiko Mitsuhashi, Yusuke Dodo, Nobuhiro Sakai, and Masamichi Takami. 2019. "Hypergravity Induces Vertebrae and Otolith Deformation in Medaka Fish." 33: 12-17.

Chatani, Masahiro, Hiroya Morimoto, Kazuhiro Takeyama, Akiko Mantoku, Naoki Tanigawa, Koji Kubota, Hiromi Suzuki, Satoko Uchida, Fumiaki Tanigaki, Masaki Shirakawa, Oleg Gusev, Vladimir Sychev, Yoshiro Takano, Takehiko Itoh, and Akira Kudo. 2016. "Acute Transcriptional Up-Regulation Specific to Osteoblasts/ Osteoclasts in Medaka Fish Immediately after Exposure to Microgravity." Scientific Reports (August): 1-14.

Enny, Alyssa, Kathleen Flaherty, Shunsuke Mori, Natalie Turner, and Tetsuya Nakamura. 2020. "Developmental Constraints on Fin Diversity." Development Growth and Differentiation 62(5): 311-25.

Ghosh, Payal, John N. Stabley, Bradley J. Behnke, Matthew R. Allen, and Michael D. Delp. 2016. "Effects of Spaceflight on the Murine Mandible: Possible Factors Mediating Skeletal Changes in Non-Weight Bearing Bones of the Head." Bone 83: 156-61.

Granet, Corinne, Alain Guignandon Laurence Vico, Christian Alexandre, and Marie-Helene Lafage-Proust.
2002. "MAP and Src Kinases Control the Induction of AP-1 Members in Response to Changes in Mechanical Environment in Osteoblastic Cells." Cellular Signalling 14(8): 679-88.

ljiri, Kenichi. 1995. "Fish Mating Experiment in Space." Biological Sciences in Space 9(1): 3-16.

Lawrence, E. A., J. Pei, C. Hammond, J. Aggleton, J. van Loon, J. Godivier, N. Nowlan, and R. Harniman. 2021. "Exposure to Hypergravity during Zebrafish Development Alters Cartilage Material Properties and Strain Distribution." Bone and Joint Research 10(2): 137-48.

Matta, Alexander, Javid Bayandor, Francine Battaglia, and Hodjat Pendar. 2019. "Effects of Fish Caudal Fin Sweep Angle and Kinematics on Thrust Production during Low-Speed Thunniform Swimming."

Mednieks, Maija I., and Arthur R. Hand. 2019. "Oral Tissue Responses to Travel in Space." Intech.

Schneider, Caroline A., Wayne S. Rasband, and Kevin W. Eliceiri. 2012. "NIH Image to ImageJ: 25 Years of Image Analysis." Nature Methods 9(7): 671-75.

Simmons, D. J., J. E. Russell, F. Winter, P. Tran Van, A. Vignery, R. Baron, G. D. Rosenberg, and W. V Walker. 1983. "Effect of Spaceflight on the Non-Weight-Bearing Bones of Rat Skeleton." The American Journal of Physiology 244(3): R319-26.

Stavnichuk, Mariya, Nicholas Mikolajewicz, Tatsuya Corlett, Martin Morris, and Svetlana V. Komarova. 2020. "A Systematic Review and Meta-Analysis of Bone Loss in Space Travelers." Npj Microgravity 6(1).

Ulbrich, Claudia, Markus Wehland, Jessica Pietsch, Ganna Aleshcheva, Petra Wise, Jack Van Loon, Nils Magnusson, Manfred Infanger, Jirka Grosse, Christoph Eilles, Alamelu Sundaresan, and Daniela Grimm. 2014. "The Impact of Simulated and Real Microgravity on Bone Cells and Mesenchymal Stem Cells." BioMed Research International 2014.

Zaitsu, Takashi, Toshiko Ohta, Hiroshi Ohshima, and Chiaki Mukai. 2014. "The Importance and Necessity of Space Dentistry." Transactions of the Japan Society for Aeronautical and Space Sciences, Aerospace Technology Japan 12(ists29):Tp_7-Tp_9.

Zhiqiang, X. I. N., and W. U. Chuijie. 2013. "Shape Optimization of the Caudal Fin of the ThreeDimensional Self-Propelled Swimming Fish." 56(2): 328-39.

This article is licensed under a Creative Commons [Attribution 4.0 International] license. https://creativecommons.org/licenses/by/4.0/ 